\title{
Alteration in Status of Olfaction amongst COVID 19 Patients: A Descriptive Study in a Dedicated COVID Hospital of Eastern India
}

https://doi.org/10.47210/bjohns.2020.v28i3.375

Monoj Mukherjee, ${ }^{1}$ Anupam Ray, ${ }^{1}$ Arpita Mohanty, ${ }^{1}$ Sekhar Ranjan Paul ${ }^{2}$

Introduction

ABSTRACT

COVID 19 patients present with symptoms of respiratory tract infection as it is caused by SARS Cov-2 which is a $\beta$ corona virus. A significant number of patients may complain of changes in olfaction either at the onset or later. The incidence, severity and recovery from anosmia/hyposmia varies in different patients. This study was done to investigate the effect of COVID 19 on olfactory dysfunction in the Indian population.

Materials \& Methods

100 patients admitted with RT-PCR positive reports for SARS Cov-2, in a dedicated COVID hospital in eastern India, were included in this study. Their olfactory function was estimated by pocket smell test (4 items) during ENT examination at bed side. Recovery from anosmia/hyposmia were noted during follow up visits.

Results

In our study $36 \%$ of patients had some alteration in smell. Out of the $36 \%, 12 \%$ had anosmia and $24 \%$ had hyposmia. Chances of complete recovery is more in patients suffering from anosmia than hyposmia.

Conclusion

Changes in smell sensation is a significant marker for screening and diagnosis of cases of COVID 19. Most of the patients recover completely.

$\underline{\text { Keywords }}$

COVID 19; SARS CoV 2; Anosmia; Hyposmia

$\mathrm{T}$ he highly transmittable Corona Virus Disease 19 (COVID-19) is caused by a $\beta$ corona virus known as Severe Acute Respiratory Syndrome corona virus-2 or SARS Cov-2. It was first reported in Wuhan, China, on 31st December, 2019 (WHO) and spread throughout the world, creating a pandemic and continues to take numerous lives daily. Human-to-human transmission is increasing at a troublesome exponential rate, which has led to steep curves of infection in many areas . ${ }^{1}$

Most of the infected people develop mild to moderate symptoms, some of them being fever, malaise and non-productive cough. ${ }^{2}$ Some people may present with changes in smell and taste sensations (e.g. anosmia and dysgeusia), sore throat, myalgia, etc. Anosmia and dysgeusia may be the presenting symptoms in a significant number of patients infected with COVID 19.

Immediate self-recognition of olfactory dysfunction is thought to be present in the most severe cases, or it is only self-identified after a prolonged latency period. ${ }^{3,4}$

1 - Department of ENT, Nil Ratan Sircar Medical College, Kolkata

2 - Department of General Medicine, Infectious Disease and Beleghata General Hospital, Kolkata

Corresponding author:

Dr Monoj Mukherjee

email: arpita.mohanty.758@gmail.com 
Temporary loss of smell and some disturbances in taste are commonly seen in patients with Viral Upper Respiratory Tract Infection through an inflammatory reaction of the nasal mucosa and the development of rhinorrhea. The most familiar agents associated being rhinovirus, parainfluenza, Epstein-Barr virus and some coronavirus 5,6. However, olfactory dysfunction linked to COVID-19 infection seems peculiar, as it is not associated with rhinorrhea.

Lechien et al. found in their study within European population that olfactory disorder may appear before the rest of the complaints in $11.8 \%$ of cases, making them very important for early detection of the disease 7.A study done by Luers et al.8 in Germany, shows that $70 \%$ of the patients suffering from COVID 19 had olfactory dysfunction. But there is scarcity of studies in the Indian population.

The objectives were to study the magnitude of changes in smell among all COVID 19 patients admitted in ID and BG Hospital, Kolkata, elicit the factors associated with the changes and estimate the median recovery time of changes in smell among the study subjects.

\section{Materials and Methods}

A descriptive type of cross sectional study was conducted in a dedicated centre for COVID-19 infected cases in Kolkata over a period of 2 months, with 100 study subjects. All of them were laboratory tested COVID positive patients, without any debilitating symptoms, who consented to participate in the study. Clearance was obtained from the institutional ethics committee.

A Pocket smell test $\{(4$ items- soap, grap, onion, gas) on basis of olfaction test of the 2013-14 US, National Health and Nutrition Examination Survey (NHANES) $\},{ }^{9}$ was used as the study tool and clinical data was prospectively collected during the ear, nose, and throat (ENT) consultation.

Small dark colour bottles of same shape and size, containing the essence were kept in front of the patient. The patient was asked to compress each nostril in turn and sniff through the other, to confirm airway patency. The test odour was then placed under one nostril while the other is compressed, and the patient was asked to take two good, but not overexuberant sniffs. He was then asked:

1. If he could smell anything

2. If he could identify the odour

If he could identify the odour, then the test was repeated in the other nostril.

After an interval to allow the odour to disperse, the test was then repeated with three other odours, and in addition he was asked

3. If he could distinguish the different odours.

After the examination, the patients were grouped into following :

Anosmia: Those who did not perceive any smell

Hyposmia: Those who perceived odour of less than four items

Normosmia: Those who could identify all the 4 items

For a good clinical history, each patient was given a proforma to fill which was later evaluated.

Patients were followed up over phone after discharge, for two months regarding full recovery of the symptoms.

Statistical analysis was done using Microsoft Excel.

\section{Results}

Data was collected from 100 patients admitted in the COVID ward of Infectious Disease and Beliaghata General Hospital, Kolkata and they were categorised. Our study included 68 males and 32 females. (Fig.1)

The patients were divided into 5 groups based on their age distribution. Out of the 100 participants, $36 \%$ ( maximum number) were in 20-30 years of age, followed by $16 \%$ in $31-40$ years, $24 \%$ in $41-50$ years, $16 \%$ in 51 60 years and $8 \%$ in more than 60 years. (Fig. 2)

Assessment of proforma filled by patients revealed that out of the 100 participants, $64 \%$ were normosmic, $24 \%$ had hyposmia and 12\% had anosmia. (Fig. 3) Most of the (10) patients of hyposmia were in the age group of 51- 60 years, and maximum number of patients with anosmia (8) were in the age group of 20- 30 years. So anosmia was more common in younger age group whereas, hyposmia was more in older age group. (Fig. 4) 


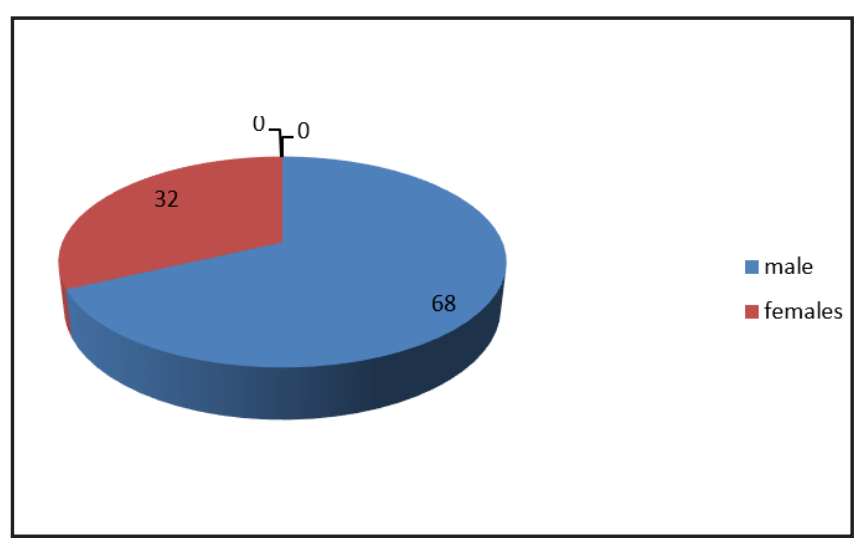

Fig. 1. Distribution of sex

Among patients with anosmia and hyposmia, maximum had onset of the alteration of smell within 1week of other symptoms, whereas the alteration of smell was the presenting feature in $33.33 \%$ of anosmic patients and $12.5 \%$ of hyposmic patients. (Table I, Fig. 5). The patients were followed up till discharge and after discharge from the hospital, over phone regarding persistence of symptoms. Maximum patients with alteration of smell improved within the 1 st month( i.e. $58.33 \%$ of patients with anosmia and $70.83 \%$ patients with hyposmia). $16.67 \%$ of the anosmia patients and $20.83 \%$ hyposmia patients had persisting dysfunction even after 2 months (Table II ).

\section{Discussion}

100 patients who were tested positive for SARS-COV 2

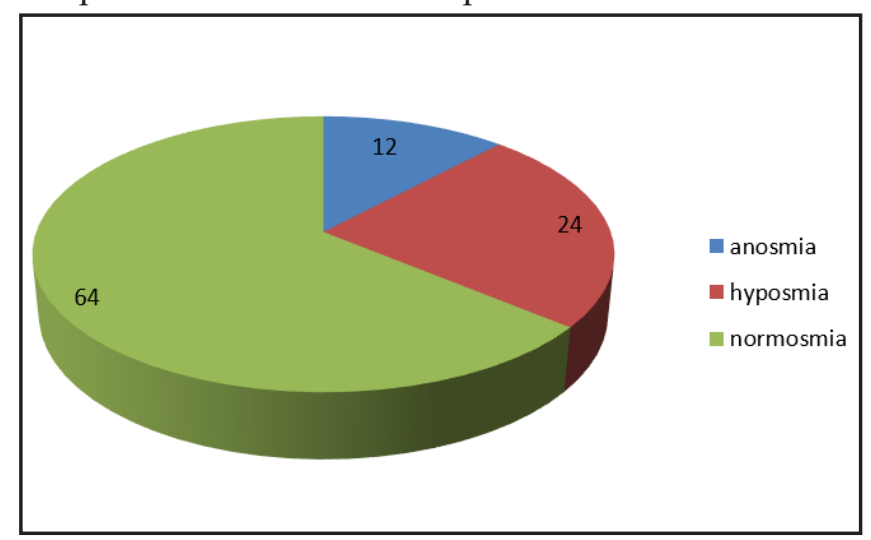

Fig. 3. Alteration of smell

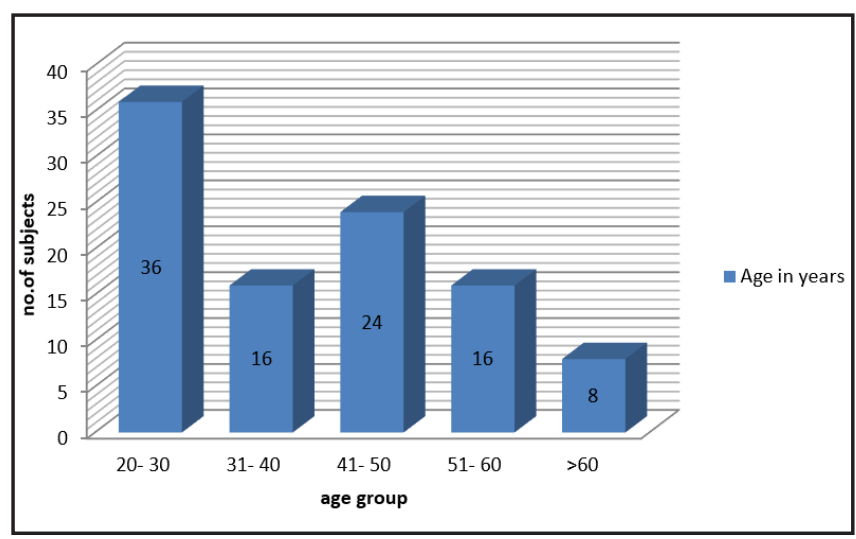

Fig. 2. Age group Distribution

by Reverse Transcriptase- Polymerase Chain Reaction test, and volunteered for the study were included in the study. Out of the 100 subjects, $68 \%$ were male and $32 \%$ females. Maximum number of patients $(36 \%)$ were in $20-30$ years of age, followed by $16 \%$ in $31-40$ years, $24 \%$ in $41-50$ years, $16 \%$ in $51-60$ years and $8 \%$ in more than 60 years. History and examination showed that $64 \%$ of them were normosmic, $24 \%$ had hyposmia and $12 \%$ had anosmia. $45 \%$ of the patients had known contact with a patient tested positive for COVID 19.

In our study $36 \%$ of patients had some alteration in smell and $13 \%$ had alteration in taste. Out of the $36 \%$, $12 \%$ had anosmia and 24\% had hyposmia ,which was confirmed by clinical history and pocket smell test.

A study by Jan C. Luers8 in Germany, which showed that $70 \%$ of the patients had olfactory dysfunction, is in contrast to our study.

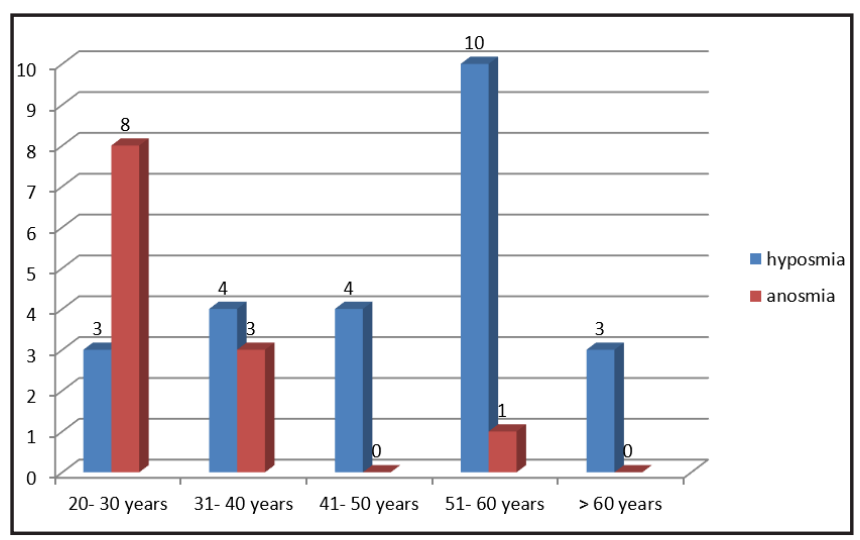

Fig. 4. Age wise distribution of patients having anosmia and hyposmia 
Table I: Onset of alteration of smell with respect to other symptoms

\begin{tabular}{|c|c|c|}
\hline ONSET OF THE ALTERATION OF SMELL & ANOSMIA (12) & HYPOSMIA(24) \\
\hline Simultaneously with other symptoms & $\mathbf{4 ( 3 3 . 3 3 \% )}$ & $\mathbf{3 ( 1 2 . 5 \% )}$ \\
\hline Within a week of other symptoms & $\mathbf{8}(66.67 \%)$ & $21(87.5 \%)$ \\
\hline
\end{tabular}

Table II: Time taken for recovery of symptoms

\begin{tabular}{|c|c|c|}
\hline RECOVERY OF SYMPTOMS & ANOSMIA (12) & HYPOSMIA(24) \\
\hline Full recovery within a month & $\mathbf{7 ( 5 8 . 3 3 \% )}$ & $\mathbf{1 7}(\mathbf{7 0 . 8 3 \% )}$ \\
\hline Recovery between 1- 2 months & $\mathbf{3 ( 2 5 \% )}$ & $\mathbf{2 ( 8 . 3 3 \% )}$ \\
\hline Persisting dysfunction after 2 months & $\mathbf{2 ( 1 6 . 6 7 \% )}$ & $\mathbf{5 ( 2 0 . 8 3 \% )}$ \\
\hline
\end{tabular}

One of the most commonly identified causes of olfactory loss is Upper Respiratory Tract Infection, accounting for $22 \%$ to $36 \%$ of cases, ${ }^{10}$ which is consistent with our study. In a study by Lechien et al, ${ }^{7}$ a total of 357 patients $(85.6 \%)$ had olfactory dysfunction related to the COVID 19 infection, among which 284 $(79.6 \%)$ patients were anosmic and 73 (20.4\%) were hyposmic . Lovato and de Filippis reviewed five articles about the clinical presentation of COVID-19 patients from China, comprising 1556 cases, with no reports of olfactory dysfunction. ${ }^{11}$ Mao et al. ${ }^{12}$ analysed the neurologic symptoms of 214 patients retrospectively in Wuhan, China, and found that $5.1 \%(n=11)$ of the patients exhibited smell impairment. The above two studies show that smell impairment is lesser in Chinese population as compared to European and American population. In a study by Khare et al, ${ }^{13}$ the percentage of COVID-19 positive individuals who were unable to either smell or identify the odorants correctly, or both, went as high as $48.9 \%$ for single odorant and $22.5 \%$ for two odorants. The difference in identification of smell and complete inability to sense the smell was

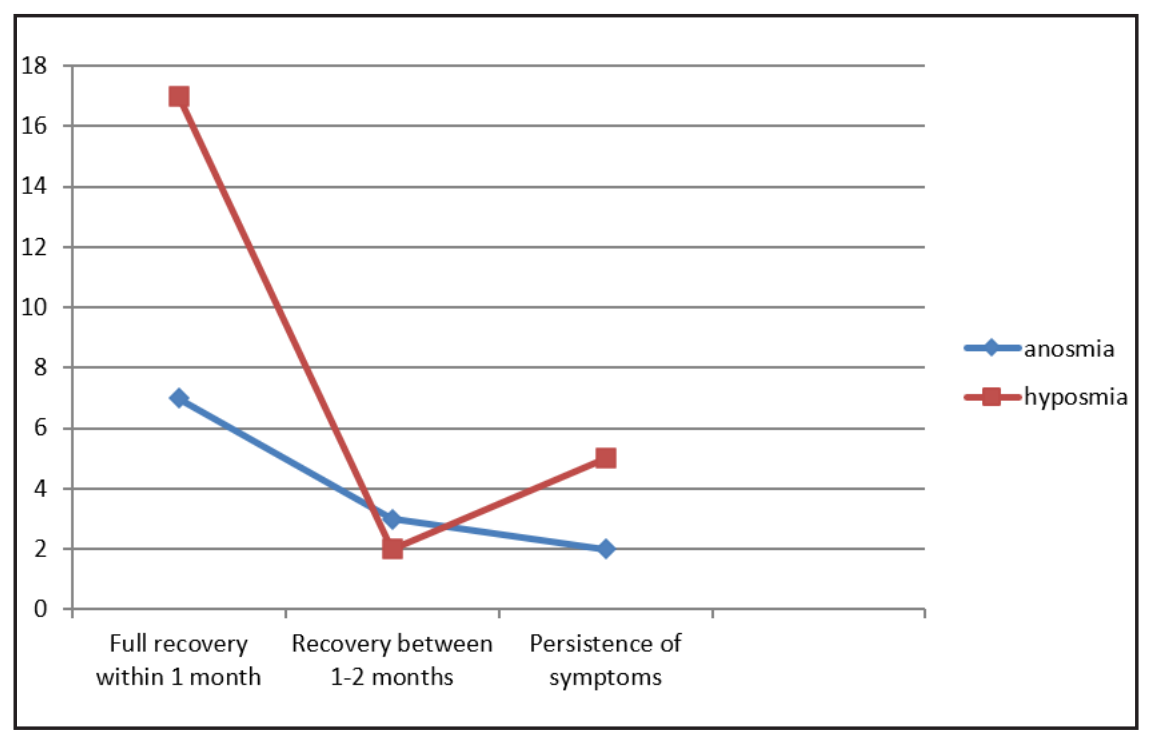

Fig. 5. maximum improvement in patients within 1 month period 
different among COVID-19 positive individuals as $38.8 \%$ were completely unable to smell a single thing and $16 \%$ individuals could not smell two odorants. In the study by Mishra et al where 74 COVID-19 patients were evaluated for presence of anosmia, it was found that 11 patients $(14.8 \%)$ had symptoms of anosmia while in controls that were COVID-19 negative, none had anosmia and only one subject had hyposmia. ${ }^{14}$ Our study is consistent with other Indian studies with respect to the prevalence of anosmia and hyposmia.

Among patients with anosmia and hyposmia, maximum had onset of the alteration of smell within 1 week after appearance of other symptoms. Alteration of smell was the presenting feature in $33.33 \%$ of anosmic patients and $12.5 \%$ of hyposmic patients.

Maximum patients with alteration of smell improved within the 1 st month, i.e. $58.33 \%$ of patients with anosmia and $70.83 \%$ patients with hyposmia showed complete improvement in the first month. $25 \%$ of anosmic patients and $8.33 \%$ of hyposmic patients had complete improvement after 1st month of treatment, whereas $16.67 \%$ of the anosmia patients and $20.83 \%$ of hyposmia patients had persisting dysfunction even after 2 months of follow up.

In a study by Lechien et al, $72.6 \%$ of these patients recovered from the olfactory dysfunction within the first 8 days following the resolution of the disease and $25.5 \%$ of patients recovered both olfactory and gustatory functions throughout the 2 weeks after the resolution of the general symptoms. ${ }^{7}$ In the study by Mishra et al. 7 out of 11 patients had regained the sense of smell 14 days following discharge and all the patients had regained their olfaction at the end of 21 days. ${ }^{14}$

Upper respiratory tract infection is one of the most commonly identified causes of olfactory loss, accounting for $22 \%$ to $36 \%$ of cases. ${ }^{10}$ Many viruses which cause olfactory dysfunction is through an inflammatory reaction of the nasal mucosa and thus also present with rhinorrhea; like rhinovirus, parainfluenza, and some coronavirus. ${ }^{5,6}$ However, olfactory dysfunction linked to COVID-19 infection is different in a sense that it is not associated with rhinorrhea.

The mechanism of action of the Novel Corona virus (SARS- $\mathrm{CoV}-2$ ), might be similar to the family of corona virus, because of the similarity in their structure. ${ }^{15}$ Coronaviruses could invade the brain via the cribriform plate close to the olfactory bulb and the olfactory epithelium. Yao et $\mathrm{a}^{16}$ have reported that the volume of the olfactory bulb is decreased in patients with postinfectious olfactory loss and is inversely related to the duration of olfactory loss. Recently Ligget et $\mathrm{al}^{17}$ described expression of the olfactory receptor family on central cortical neurons, vascular smooth muscle, and upper and lower airway epithelium. Fodoulian et al, identified ACE 2 and TMPRSS2 receptors in the olfactory sustentacular cells, horizontal, basal and microvascular cells. ${ }^{18}$ The spike protein $(\mathrm{S})$ on the surface of SARS-CoV-2 virus plays an essential role of attachment of the virus particle on ACE 2 receptor and its transneuronal spread.

A scarcity of acute phase advanced neuroimaging studies, difficulties in obtaining histopathological tissue specimens, and an absence of viral cultures of infected olfactory neuroepithelium compound the difficulties in studying this phenomenon.

\section{Conclusion}

Our study shows that alteration of smell can be the sole presenting symptom in patients with COVID 19, which can be included as a criteria for isolation of the subjects to prevent spread of the infection.

There is also a significant variance in the occurrence of olfactory dysfunction among COVID 19 patients, rates being lesser in the Indian population compared to the American and European populations.

Young patients do suffer from anosmia more than the aged patients. There is more probability of complete recovery from anosmia than hyposmia.

Another important finding is that some patients can have a persistent loss of smell following infection with COVID 19, which has to be explained to the patients on appearance of the symptoms.

The possibility of COVID 19 infection should be considered clinically in any patient presenting with change in smell or taste disorder with new onset of pyrexia with a positive history of contact with COVID 19 patients and / or travel prior to 14 days to a location 
where there is community transmission of SARSCOV-2 (COVID 19).

\section{References}

1. Wu YC, Chen CS, Chan YJ. Overview of The 2019 Novel Coronavirus (2019-nCoV): The Pathogen of Severe Specific Contagious Pneumonia (SSCP). J Chin Med Assoc. 2020. doi: 10.1097/JCMA.0000000000000270.

2. Huang $\mathrm{C}$,Wang $\mathrm{Y}$, Li X, et al. Clinical features of patients infected with 2019 novel coronavirus in Wuhan, China. Lancet. 2020;395(10223):497-506doi:10.1016/S0140-6736(20)301835

3. Xydakis MS, Mulligan LP, Smith AB, et al. Olfactory impairment and traumatic brain injury in blast-injured combat troops: a cohort study. Neurology 2015; 84: 1559-67.

4. Xydakis MS, Belluscio L. Detection of neurodegenerative disease using olfaction. Lancet Neurology 2017; 16: 415-16.

5. Suzuki M, Saito K, Min WP, Vladau C, Toida K, Itoh H, Murakami S. Identification of viruses in patients with postviral olfactory dysfunction. Laryngoscope. 2007; 117(2):272-7.

6. Van Riel D, Verdijk R, Kuiken T. The olfactory nerve: a shortcut for influenza and other viral diseases into the central nervous system. J Pathol. 2015; 235(2):277-87. doi: 10.1002/path.4461.

7. Jerome R. Lechien et al,Olfactory and Gustatory Dysfunctions as a Clinical Presentation of Mild to Moderate forms of the Coronavirus Disease (COVID-19): A Multicenter European Study, Email: Jerome.Lechien@umons.ac.be

8. Jan C. Luers, MD; Alexander C. Rokohl, MD; NiklasLoreck; Philomena A. Wawer Matos; Max Augustin ; Felix Dewald, MD; Florian Klein, MD; Clara Lehmann MD; Ludwig M. Heindl, MD, Olfactory and Gustatory Dysfunction in Coronavirus Disease 19 (COVID-19), Oxford University Press for the Infectious Diseases Society of America, 2020.

9. Smell and Taste Disorders: Diagnosis - Medical Clinical Policy Bulletins $\mid$ Aetna.
10. Seiden AM, Duncan HJ. The diagnosis of a conductive olfactory loss. Laryngoscope. 2001;111(1):9-14. doi:10.1097/00005537200101000-00002.

11. Lovato A, de Filippis C. Clinical presentation of COVID-19: a systematic review focusing on upper airway symptoms. Ear Nose Throat J 2020. https://doi.org/10.1177/0145561320920762. [145561320920762, Apr 13. [Epub ahead of print]].

12. Mao L, Jin H, Wang M, Hu Y, Chen S, He Q, et al. Neurologic manifestations of hospitalized patients with coronavirus disease 2019 in Wuhan, China. JAMA Neurol 2020. https:// doi.org/10.1001/jamaneurol.2020.1127. [Apr 10. [Epub ahead of print]]

13. Pragyanshu Khare1, Atul Munish Chander1 et al , Utility of Olfactory test as screening tool for COVID-19: A pilot Study; medRxiv preprint doi: https://doi. org/10.1101/2020.09.03.20187294

14. Prasun Mishra, Vishwanath Gowda et ,Prevalence of New Onset Anosmia in COVID-19 Patients: Is The Trend Different Between European and Indian Population? al Indian J Otolaryngol Head Neck Surg, https://doi.org/10.1007/s12070020-01986-8.

15. Li YC, BaiWZ, Hashikawa T. The neuroinvasive potential of SARS-CoV-2 may play a role in the respiratory failure of COVID-19 patients [published online,February 27, 2020]. J Med Virol. doi:10.1002/jmv.25728.

16. Yao L, Yi X, Pinto JM, et al. Olfactory cortex and olfactory bulb volume alterations in patients with post-infectious olfactory loss. Brain Imaging Behav.2018;12(5):1355-1362. doi:10.1007/s11682-017-9807-7

17. An SS, Liggett SB. Taste and smell GPCRs in the lung: evidence for a previously unrecognized widespread chemosensory system. Cell Signal. 2018;41:82-88. doi:10.1016/j.cellsig.2017.02.002.

18. Leon Fodoulin, Joel Tuberosa, Daniel Rossier, et al,SARS$\mathrm{CoV}-2$ receptor and entry genes areexpressed by sustentacular cells in human olfactory neuroepithelium, https://doi. org/10.1101/2020. 


\section{PROFORMA}

1. Age:

2. Sex:

3. Address:

4. Chief complaints:

5. History of present illness:

a. When did the symptom of reduced sensation of smell start (with reference to contact history/ first symptom/ test positive)

b. If there was a complete or partial loss of smell

c. Did the symptoms recover with time?

d. If so, how much time did it take to recover?

6. History of past illness:

a. Travel History: (International / Other state)

b. History of contact with COVID positive patients: Yes/ No

c. Comorbidities: Diabetes Mellitus/ Hypertension/ COPD/ SARI

d. Previous history of Chronic rhinosinusitis / Nasal polyposis/ Allergic rhinosinusitis, nasal surgery.

e. Past history of trauma to the nose/ head injury

f. History of any Neurodegenerative disease (eg Parkinson's disease, Multiple myeloma)

7. Examination of the nose:
a. External nose
b. Vestibule
c. Anterior rhinoscopy (without a speculum): to see if there is any gross obstruction.

8. Olfactory examination: (with Pocket smell test ( 4 items- soap, grape, onion, natural gas):
a. If he can smell anything : (yes/ no)
b. If he can identify the odour: (yes/ no)
c. If he can distinguish the different odours: (yes/ no) 